\title{
Respiratory resistance measured by an airflow perturbation device
}

\author{
Christopher G Lausted and Arthur T Johnson† \\ Biological Resources Engineering, University of Maryland, College Park, MD 20742-5711, USA
}

Received 14 April 1998, in final form 8 September 1998

\begin{abstract}
The airflow perturbation device (APD) is an instrument for the measurement of respiratory resistance. The APD is small, lightweight fast and requires no special breathing manoeuvres. Airflow perturbation determines resistance by superimposing a periodic signal onto spontaneous breathing with a variable resistance device. Respiratory impedance is the ratio of magnitude of pressure perturbation to magnitude of flow perturbation, and respiratory resistance is the in-phase portion of respiratory impedance. The APD was tested to determine its responses to repeated resistance measurements and to changes in resistance. A mechanical model test showed that the APD could detect increased resistance levels, but overestimated resistance when resistance increased with flow. Tests with human subjects showed that the APD gave results consistent from day to day, was able to detect added resistances and gave resistance values correlated with airway resistance values obtained by body plethysmography. Accelerometers placed on the chests of the subjects showed that perturbations extend to the chest surface. Thus, the APD must measure total respiratory resistance.
\end{abstract}

Keywords: respiration, resistance

\section{Introduction}

Respiratory resistance is a measurement of clinical and physiological interest. Increased resistance is clinically related to an assortment of respiratory diseases such as asthma, bronchitis, pneumonia, emphysema and other obstructive disorders. Resistance measurement is useful in evaluating the respiratory effects of bronchoconstrictive and bronchodilatory drugs, as well as airborne contaminants and naturally occurring particulates. Despite the usefulness of respiratory resistance measurement, there is still no instrument that is reasonably inexpensive, easily used and widely applicable.

Several techniques are available to measure various portions of respiratory resistance. Measurements taken are either of airway resistance, pulmonary resistance or total respiratory resistance. Pulmonary resistance is the sum of airway and lung tissue resistance. Total respiratory resistance is the sum of pulmonary and chest wall resistances. Among non-invasive methods of measurement, the technique of forced oscillation (Barnas et al 1987, Suki et al 1989, Michaelson et al 1975, Nagels et al 1980) measures total respiratory resistance, but it requires a great deal of expertise and subject cooperation. Whole-body plethysmography (DuBois et al 1956, Stanescu 1991, Teculescu et al 1982) measures airway resistance, but it requires a large apparatus that is not portable or easy to use. Both techniques are quite expensive. Other techniques are invasive, such as the insertion of an oesophageal balloon directly into the subject for the measurement of pulmonary resistance.

† E-mail address: aj16@umail.umd.edu

0967-3334/99/010021+15\$19.50 ๑ 1999 IOP Publishing Ltd 
Airflow perturbation techniques were proposed by several groups (Sobol 1970a, b, 1971, Johnson et al 1974, Kures 1974. Schmid-Schoenbein and Fung 1978) in the 1970s as a simple way of obtaining respiratory resistance. The APD was intended to be a simple, economical, non-invasive device (Johnson et al 1974) for the measurement airway resistance (Johnson and Lin 1983a), and has been tested on pigs, sheep and humans (Johnson and Lin 1983b, Johnson et al 1984, Lehtola 1986). However, APD measurements made at that time required manual analysis rather than computer automation and thus a great number of questions remained about the accuracy, reliability and meaning of the measurements (Lin et al 1985).

In this paper we describe an improved version of the APD and results from testing of the device (Lausted 1997). Features of this device are:

(1) It offers non-invasive, direct measure of respiratory resistance.

(2) It is lightweight, portable and inexpensive.

(3) Measurements are made in less than $1 \mathrm{~min}$ and are continuously updated.

(4) Spontaneous breathing is measured - does not require conscious or cooperative patients.

(5) There is separate measurement of inhalation and exhalation resistances.

(6) It requires no special skill to use.

(7) Results are highly reproducible with low variation.

(8) Measurements are sensitive to changes in resistance.

\section{Device description}

The APD consists of a perturbation mechanism, a perturbation mechanism power supply, a signal conditioning circuit housing and a data acquisition computer (figure 1). The perturbation mechanism (figure 2) is built around a Fleish \#2 pneumotachograph (OEM Medical, Richmond. VA) with an attached differential pressure transducer used to measure flow. A small hole in the proximal flange of the pneumotachograph serves as a mouth pressure tap. It is connected to a second pressure transducer that measures mouth pressure relative to the atmosphere. Both transducers are Validyne (Northridge, CA) model DP-15. Airflow is perturbed by a partially screened motor-driven wheel mounted perpendicular to the pneumotachograph flow path. The wheel is divided into segments and two of these are covered with wire screen. Two perturbations are induced with each revolution of the wheel. The dead volume of the device is the dead volume of the pneumotachograph, $37 \mathrm{ml}$.

The perturbation mechanism power supply provides $2.5 \mathrm{~V}$ a.c. to the pneumotachograph heater and $9.0 \mathrm{~V}$ d.c. to the screen motor. The signal conditioning circuit consists of a Validyne CD 72-2 dual demodulator that provides excitation to the transducers and amplification of their output. Output of the CD72-2 ranges from -10 to $+10 \mathrm{~V}$ so 2:1 voltage dividers were added to avoid saturating -5 to $+5 \mathrm{~V} \mathrm{~A} / \mathrm{D}$ converters housed in the notebook computer. Software for the APD was written in the Visual Basic for Applications (VBA) macro language used with the Microsoft Excel 4.0 spreadsheet application. Although VBA is a high-level programming language and slow in execution, a $100 \mathrm{MHz}$ PC with a 586 processor is sufficient to carry out all operations described here in real time. The software logs pressure and flow data from the perturbation mechanism, calculates a 'virtual data', and calculates and displays resistance in real time. Optionally, fast Fourier transforms of $5 \mathrm{~s}$ intervals of pressure and flow data are available, also in real time.

The APD works in a manner similar to both the forced oscillation and the flow interruption techniques. The forced oscillation technique involves inducing a pressure oscillation at the mouth and measuring the resulting flow oscillation. Respiratory impedance is the magnitude of pressure oscillation divided by the magnitude of flow oscillation. Respiratory resistance is the in-phase portion of respiratory impedance. Flow interruption involves rapidly occluding flow at the mouth. Assuming mouth pressure during the occlusion rises to equal alveolar pressure before the occlusion, airway resistance is the ratio of post-occlusion mouth pressure to pre-occlusion flow, As with these two techniques, the APD applies a signal at the mouth and measures changes in mouth pressure and flow. Passage of the screened segment of the wheel in front of the flow path causes a decrease of airflow and an increase of mouth pressure compared with the unperturbed signal. Resistance $\left(R_{\text {apd }}\right)$ is the ratio of mouth pressure 


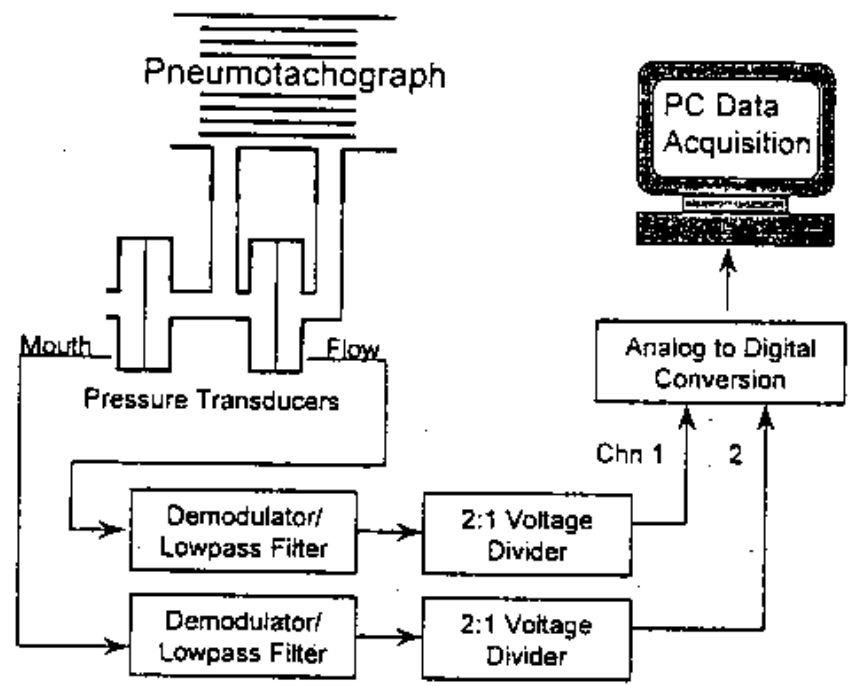

Figure 1. Diagram of the APD system.

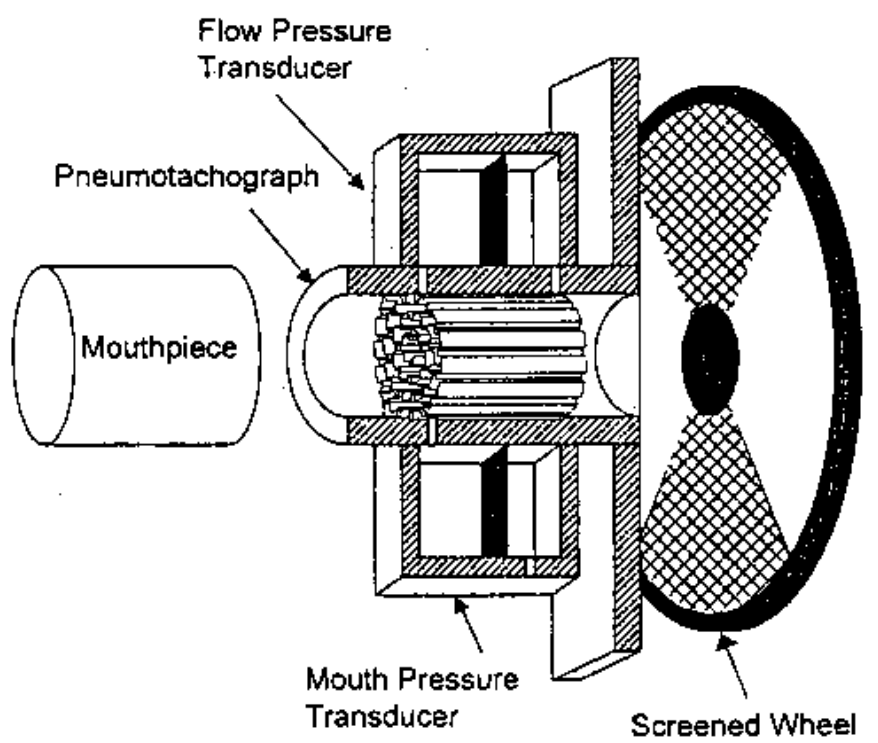

Figure 2. Cut-away diagram of airflow perturbation mechanism. The screened wheel rotates, allowing screened segments to briefly slow, or perturb, airflow passing through the pneumotachograph. Pressure transducers monitor mouth pressure and flow. 
perturbation magnitude $(\Delta p)$ to flow perturbation magnitude $\left(\Delta V^{\prime}\right)$ taken at the same time $\left(t_{0}\right)$. Typical pressure and flow perturbations are shown in figure 3. Perturbation magnitudes $\Delta p$ and $\Delta V^{\prime}$ are the respective differences between the actual signals and those that would have existed had the perturbation not occurred (called the virtual data). Time $t_{0}$ is chosen from the sample with maximum $\Delta V^{\prime}$, where volume acceleration is zero, in order to eliminate mass inertia effects. High-frequency perturbations of small magnitude are used to minimize compliance effects. Therefore, $R_{\text {apd }}$ can be considered as a resistance and not an impedance.

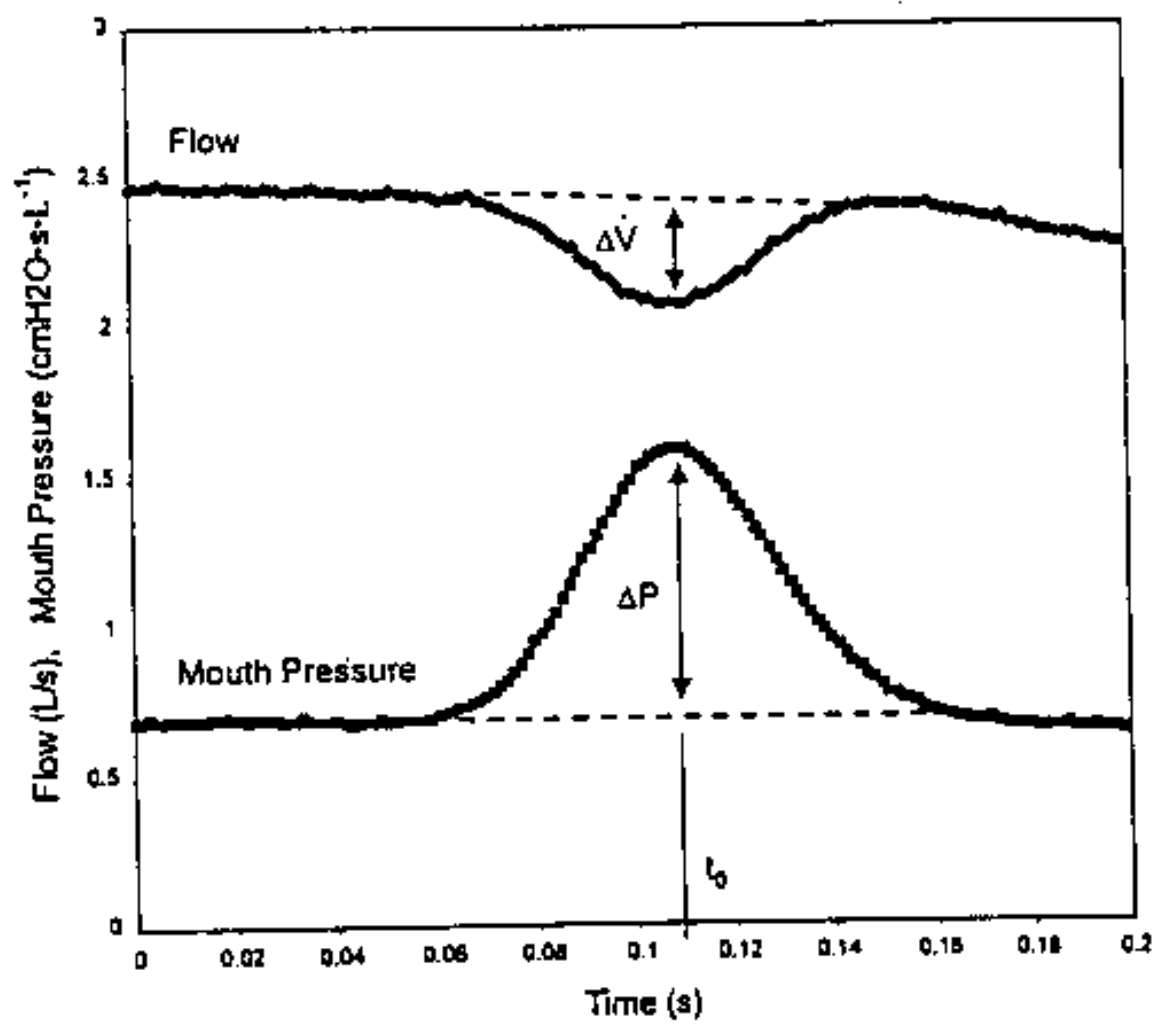

Figure 3. A typical perturbation induced by the APD.

Virtual data are obtained by linearly interpolating between the point immediately preceding the perturbation and the point immediately following the perturbation.

\section{Methods}

Seven tests were conducted to indicate the nature of APD measurements These are summarized in table 1.

\subsection{Mechanical model tests}

A mechanical analogue to the human respiratory system (figure 4) was constructed from a plastic pipe containing steel wool and connected to a large weather balloon. Pressure inside the balloon (analogous to alveolar pressure) was measured at a stagnation point to avoid velocity-related errors. Resistance of the pipe and steel wool could be measured as the pressure difference between the balloon and the mouth pressure tap.

Testing began by filling the weather balloon to approximately $35 \mathrm{~cm}$ diameter. Then the balloon was allowed to deflate. The APD motor was activated and ran at 6.7 perturbations per second, which allowed approximately 25 to 30 estimates of $R_{\text {apd. }}$. 
Table I. Summary of tests conducted with he APD.

No of subjects

Purpose

Description

\begin{tabular}{lll}
\hline Mechanical model resistance & 4 mechanical models & $\begin{array}{l}\text { Does APD correctly measure airway resistance? Are } \\
\text { corrections needed for flow dependence? }\end{array}$
\end{tabular}

APD connected to each mechanical model and run. APD resistance calculated by two methods: assuming constant resistance and assuming flow-dependent resistance

Chest wall perturbations

1 volunteer

Frequency dependence

4 volunteers

Measurements repeated over time

Sensitivity to changing resis- 6 volunteers ance: low lung volume

Sensitivity to changing resis- 10 volunteers tance: added resistors

Plethysmography

21 volunteers
Does APD measure total respiratory resistance of human subjects?

Do measurements change with wheel rotation speed?

Are APD measurements consistent over time?

Is APD sensitive to increased (internal) resistance?

Is APD sensitive to increased (external) resistance?

Is APD resistance correlated to plethysmographic resistance?
Accelerometers placed on chest wall. Detection of perturbation frequencies indicate presence of perturbations on chest wall which indicate APD measurement applies to total respiratory system

Three perturbation frequencies provided by use of three wheel rotation speeds

Measurements taken 5 times within 15 min; 3 times within three days; 3 times within 3 weeks

Measurements taken with subjects breathing in normal volume range and with same subjects breathing in decreased volume range. Decreased lung volume results in increased airway resistance

Resistors placed in series between subject and APD.

Response to added resistance measured

Subjects tested with both APD/ and body plethysmograph 


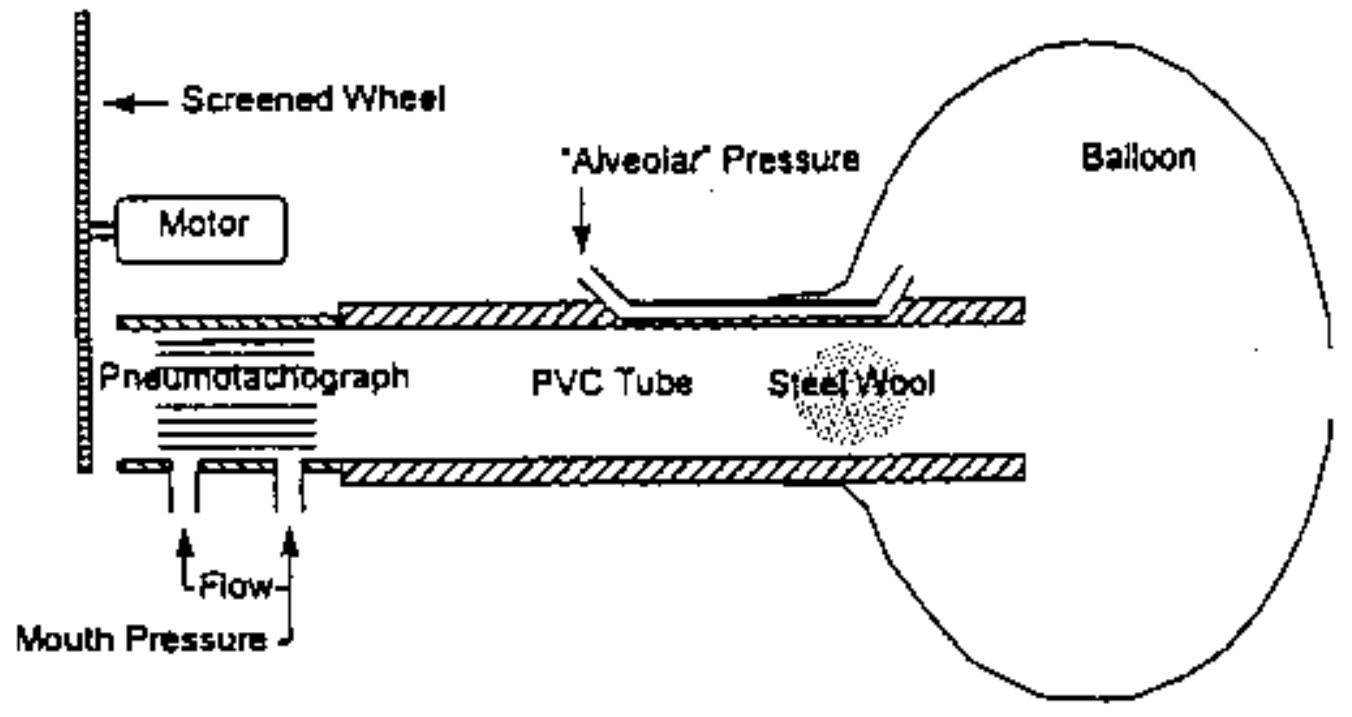

Figure 4. Mechanical respiratory system model with APD attached.

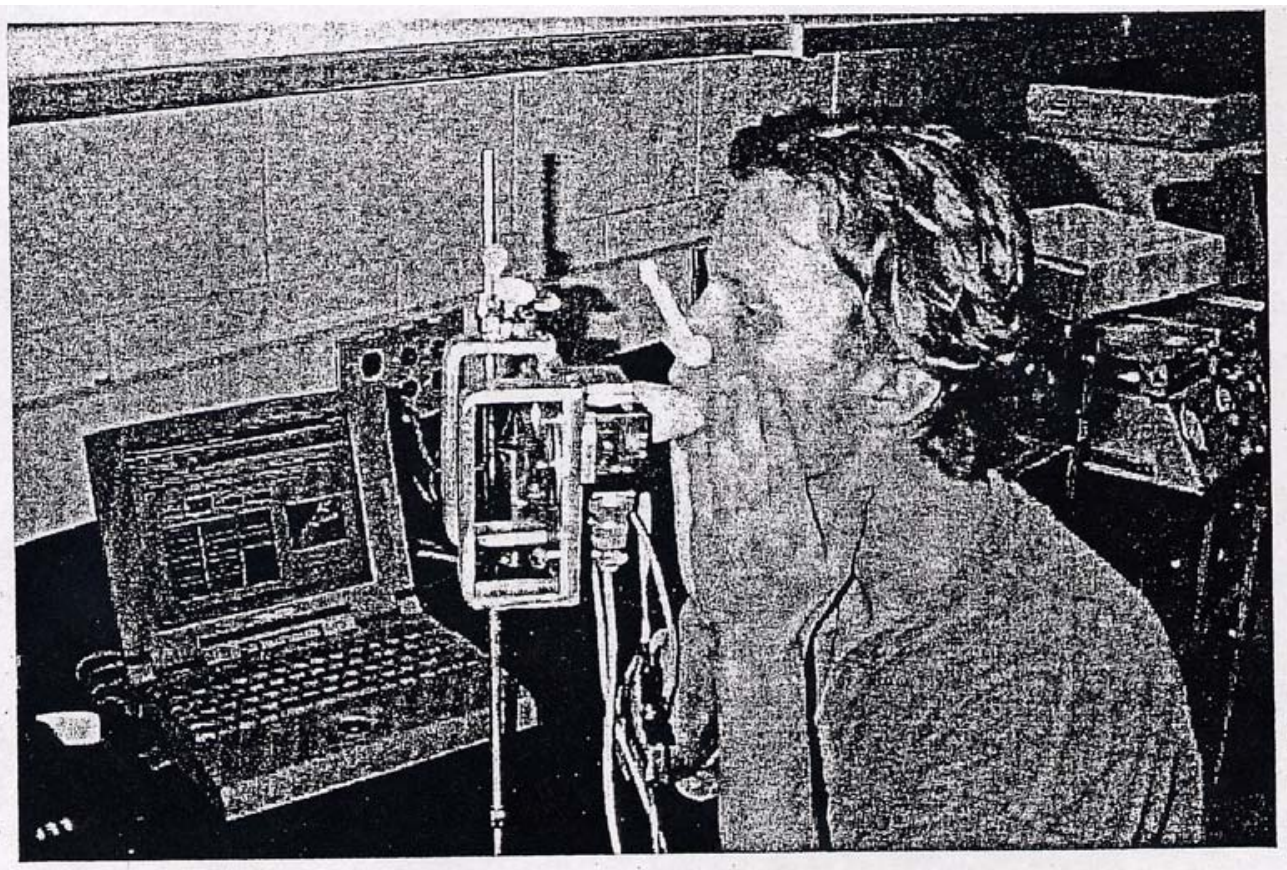

Figure 5. Usage of the APD. 


\subsection{Human subject testing with the APD}

Approval for human subject testing was obtained from the University of Maryland Institutional Review Board. All subjects tested were healthy adults with no history of respiratory disease recruited from the University of Maryland area. Subjects completed an informal consent form and brief health history questionnaire. Subjects were seated with the back and neck straight. The APD was mounted on a ring stand and the height adjusted for each individual. The subject inhaled and exhaled through the APD via mouthpiece. Nose clips were used. The subject firmly supported the cheeks with the hands (figure 5). This subject was instructed to keep the glottis open and the tongue still, in its normal position. Except where noted, the subject was instructed to breathe normally. Except where noted, the APD was operated with a wheel speed equivalent to 6.7 perturbations per second. Subjects breathed through the APD until 100 inspiratory and 100 expiratory perturbations were obtained. This took approximately $1 \mathrm{~min}$. $R_{\text {apd }}$ was calculated for each perturbation. The $R_{\text {apd }}$ values were used to calculate average inspiratory resistance $\left(R_{\text {avg,in }}\right)$, average expiratory resistance $\left(R_{\text {avg,ex }}\right)$, and overall average resistance $\left(R_{\text {avg }}\right)$. If the standard deviation of the $R_{\text {apd }}$ values were noted to be unusually high, the test was repeated. This would happen if excessive electrical noise was present in the power supply or if the subject were to hiccup, cough or close the glottis during the measurement.

3.2.1. Detection of perturbations on the chest wall. The APD has in some cases been previously described as measuring airway resistance rather than respiratory resistance. For this to be the case, the perturbations would need to be dampened out prior to reaching the chest wall. A test was conducted to detect the presence of perturbations on the chest wail. Acceleration measurements were taken on the chest wall of a subject while breathing through the APD. Acceleration was measured by a Bruel and Kjaer (Marlborough, MA) type 2035 signal analyser unit and reported as a spectral distribution in decibels from 0 to $50 \mathrm{~Hz}$. Bruel and Kjaer type 4393 accelerometers have a typical acoustic sensitivity of $0.04 \mathrm{~m} \mathrm{~s}^{-2}$ at $150 \mathrm{~dB}$ from 2 to $100 \mathrm{~Hz}$ and weigh $2.2 \mathrm{~g}$. Three accelerometers were taped to the chest wall: one centred on the right pectoral $4 \mathrm{~cm}$ from the sternum, another $5 \mathrm{~cm}$ below it, and a third $2 \mathrm{~cm}$ right of the seventh dorsal vertebra. The subject was instructed to breathe normally through the APD while wearing the nose clip and firmly supporting the cheeks. Data were collected for $15 \mathrm{~s}$.

3.2.2. Perturbation frequency dependence. The APD was tested for perturbation frequency dependence. The perturbation mechanism features a voltage-controlled screen wheel motor. Faster wheel speeds are desirable since they result in a shorter perturbation duration and a greater number of $R_{\text {apd }}$ samples. However since forced oscillation data (Barnas et al 1987) indicate a frequency dependence to respiratory resistance, it needed to be determined that $R_{\text {apd }}$ remains constant over the range of possible APD wheel speeds. Measurements of $\left(R_{\text {avg,in }}\right)$ and $\left(R_{\text {avg,ex }}\right)$ were taken at each screen wheel rotational speed of 2.2, 4.4, and 6.7 revolutions per second. The APD power supply was not capable of higher wheel speeds. The test was repeated for three subjects.

3.2.3. Monitoring APD resistance over time. The APD was tested for short-term (several minutes) and long-term (several weeks) reproducibility. First, five measurements $R_{\text {avg }}$ of were taken from four subjects over the course of 15 min. Subjects removed mouthpieces and noseclips between measurements. Next, three measurements of $R_{\text {avg }}$ were taken from six subjects over three days - one each day. Finally three measurements were taken from seven subjects over three weeks - approximately one week between each measurement.

3.2.4. Sensitivity to changing resistance. Two experiments were conducted to test the sensitivity of the APD to varying resistance. The first two experiments compared $R_{\text {avg }}$ of subjects during normal breathing with $R_{\text {avg }}$ of the same subjects breathing at decreased lung volumes. Airway resistance generally increases at lower lung volumes and so does respiratory resistance. Six subjects were tested. They were instructed in the definitions of functional residual capacity (FRC), residual volume $\left(V_{\text {res }}\right)$ and tidal volume $\left(V_{t}\right)$. FRC is average end-expiratory volume of the lungs during normal breathing; $V_{\text {res }}$ is the lung volume remaining at the end of a maximum exhalation effort; and $V_{t}$ is the average stroke volume of the lungs during normal breathing. Then they were instructed to perform two breathing manoeuvres. They were first told to breathe normally so that expiration would terminate at FRC and inspiration would terminate at FRC plus $V_{t}$. They were next told to breathe such that expiration would terminate at $V_{\text {res }}$ and inspiration would terminate at FRC. Plow and breathing frequency were to be kept as similar to normal breathing as possible. $R_{\text {avg }}$ was measured for both manoeuvres. 
The second experiment tested the APD's responsiveness to changing resistance by the insertion of flow resistors (Fleish \#2 pneumotachographs) between the subjects and the APD. Zero, one, two and three pneumotachographs were placed between the subjects' mouths and the APD. $R_{\text {avg }}$ was measured for each resistance level. The order of added pneumotachographs was $0,1,2,3$ in five subjects and 3,2,1,0 in five subjects.

3.2.5. Plethysmographic testing. Eighteen subjects were tested by whole-body plethysmography and by the APD. Subjects were tested in the Collins body plethysmograph. At least six measurements of airway resistance $\left(R_{a w}\right)$ and thoracic gas volume $\left(V_{t g}\right)$ were taken. Resistance was calculated by Collins DSPLUS software as the slope of the chord between pressure-flow data at -0.5 and $+0.51 \mathrm{~s}^{-1}$. Any measurements where $R_{a w}$ or $V_{t g}$ varied from all other subject measurements by more than $35 \%$ were discarded as outliers. The panting manoeuvre was conducted at $2 \mathrm{~Hz}$ with the aid of a metronome, Subjects were also tested to determine $R_{\text {avg. }}$. For ten subjects, the $R_{a w}$ test preceded the $R_{\text {avg }}$ test. For the remaining eight subjects, the $R_{\text {avg }}$ test preceded the $R_{a w}$ test. Tests were conducted within 10 min of each other.

\section{Results}

\subsection{Mechanical model testing}

The pressure/flow characteristics of the four mechanical model configurations were found by direct measurement to be distinctly parabolic. These could well be described by the Rohrer equation (Johnson 1986): $R=K_{1}+K_{2} V^{\prime}$. Values for $K_{l}$ and $K_{2}$ were found by least squares regression and all four curve-fit parabolas correlated with the actual data by greater than $99.9 \%$.

The presence of a positive $K_{2}$ would lead to overestimates of $R_{\text {apd }}$ compared with actual resistance (Johnson 1986, Johnson and Milano 1987). An estimate of pressure drop across the model ( $\left.p_{\text {apd }}\right)$ was calculated for each $R_{\text {apd }}$ by multiplying $R_{\text {apd }}$ by the virtual flow at the time of perturbation. The values of $p_{\text {apd }}$ for one of the model configurations (symbols) are shown along with actual pressure drop (full curve) in figure 6. The $p_{\text {apd }}$ values are consistently higher than the actual pressure, as would be expected in the presence of a positive $K_{2}$. These results indicate that if there is a large dependence of resistance on flow, then data analysis procedures have to be modified to account for this dependence. This can be done (Johnson 1986, Johnson and Milano 1987, Lausted 1997), but requires an accurate model of the form for the flow dependence. Assuming the Rohrer model given previously, $R_{\text {apd }}$ can be obtained to overestimate actual resistance by no more than $5 \%$. However, real-time estimates of $R_{\text {apd, }}$ correcting for flow dependence, cannot be made at present. Fortunately, measurements made on human subjects showed none of this flow dependence.

\subsection{Perturbations on the chest wall}

Measurements of acceleration taken on the external surface of the chest wall indicate the presence of APD perturbations. Row perturbations appeared at all three sites monitored. The full curve in figure 7 displays the discrete Fourier transform of flow measured by the APD pneumotachograph during periodic flow perturbations. Peaks occur just around the primary frequency of $6.7 \mathrm{~Hz}$ and at each of its harmonics. The data points show the spectral distribution of vibration experienced by accelerometers attached to the outside of the chest wall. Increased vibration is also present at $6.7 \mathrm{~Hz}$ and each of its harmonics. These findings indicate that the APD is measuring respiratory resistance and not merely airway resistance or pulmonary (airway plus lung tissue) resistance.

\subsection{Perturbation frequency results}

The APD produced resistance measurements that did not change noticeably with screen wheel speed (figure 8). The application of standard repeated measures ANOVA indicated that resistance measurements were not statistically different at the $\alpha=0.05$ level for the three wheel rotation speeds tested.

\subsection{Resistance over time}

The average standard deviation of repeated resistance measurements taken every $15 \mathrm{~min}$ are 0.09 and 0.10 , and are less than $4 \%$ of the average. This is a very small variation, indicating that APD measurements are consistent over short time intervals. Estimates of respiratory resistance also appear to be reproducible from day to day, with the average coefficient of variation found to be $1.8 \%$. Why the coefficient of variation from day to day is less than the variation present within a few hours is unclear. There may be no real difference, as both vibrations are small. It is 
possible that the subjects subconsciously change their breathing technique when asked to repeat the experiment. However, there was no change in breathing frequencies as average flow was carefully monitored. The ranking of subjects by $R_{\text {avg }}$ did not change over the course of the three measurements. The amount of variation was different for each subject: one subject exhibited almost no change whatsoever, less than the $3.3 \%$ variation expected to be present in measuring $R_{\text {avg }}$, another subject changed the most, dropping from 2.91 to $2.64 \mathrm{cmH}_{2} \mathrm{O} \mathrm{s}{ }^{-1}$ during the test.

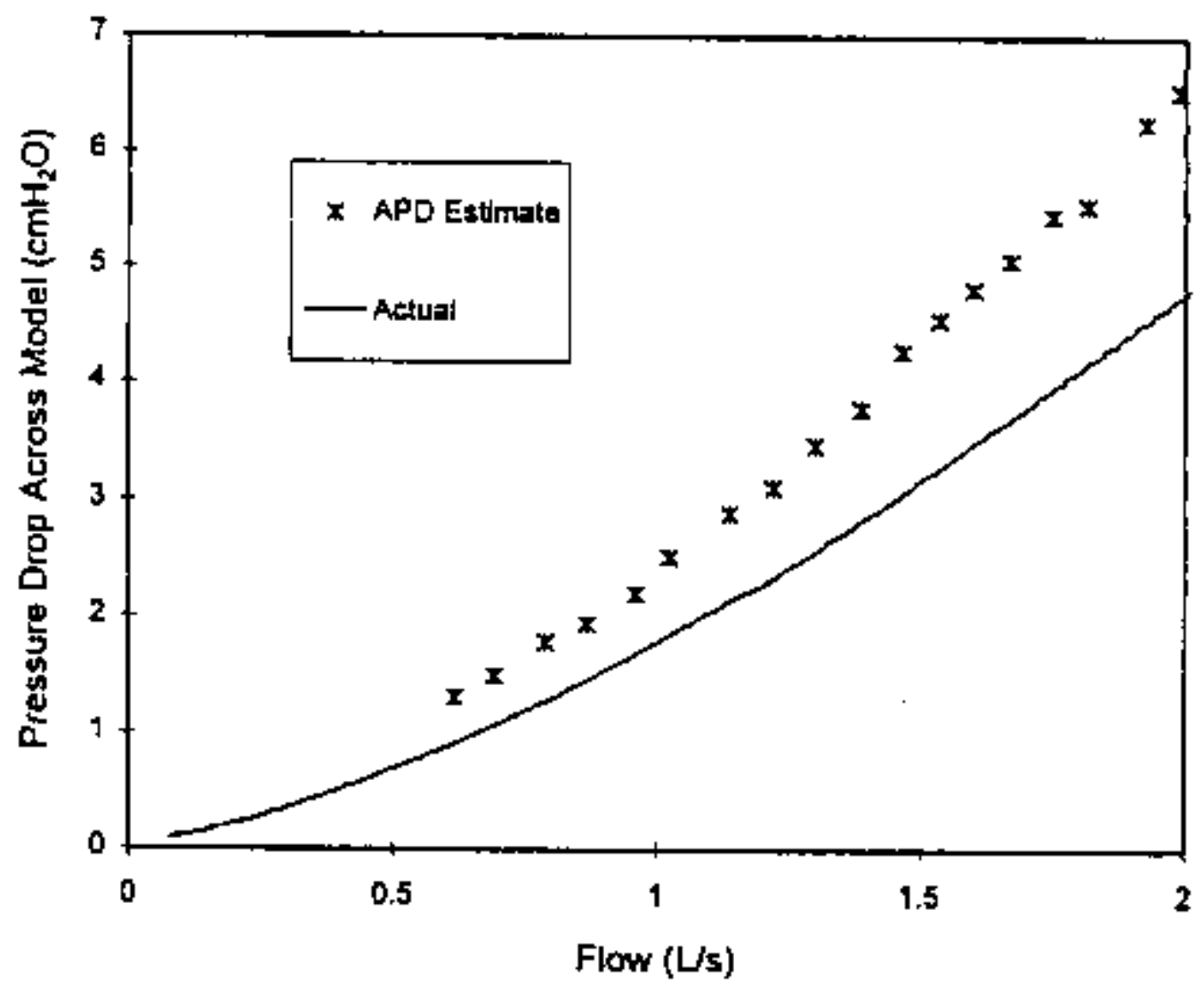

Figure 6. PIot of actual and APD-estimated mechanical model pressure/flow characteristics.

When resistance was measured once per week, an average coefficient of variation of $7.2 \%$, was present in $R_{\text {avg }}$. Subject resistance may have, in fact, changed over this time. For example, one subject began a regular programme of running between the first and the second weekly measurement. Factors such as exercise history, airborne dust or pollen, and minor illness were not carefully monitored in this experiment.

\subsection{Detection of increased resistance}

$R_{\text {avg,in }}$ and $R_{\text {avg,ex }}$ were obtained for six subjects at normal and low-volume breathing. Application of the Student's paired $t$-test to two sets of data indicate a significant difference at the $\alpha=0.05$ level. $R_{\text {avg,in }}$ was higher for lowvolume breathing than for normal breathing $(p=0.0108)$, as was $R_{\text {avg,ex }}(p=0.0068)$. This indicates that the APD is able to detect internal airway constriction.

Adding pneumotachographs between the mouth and the instrument increased measured $R_{\text {apd }}$ in each of the 10 subjects tested. Figure 9 shows $R_{\text {apd,ex }}$ plotted versus the resistance of the external pneumotachographs at the average test flow. Lines connect data points collected from each subject. Similar results were obtained for $R_{\text {avg,in. }}$ Linear regression was performed for each subject and the results averaged. The average resistance-resistance slope was $0.86 \pm 0.10$ for expiration and $0.73 \pm 0.14$ for inspiration. 


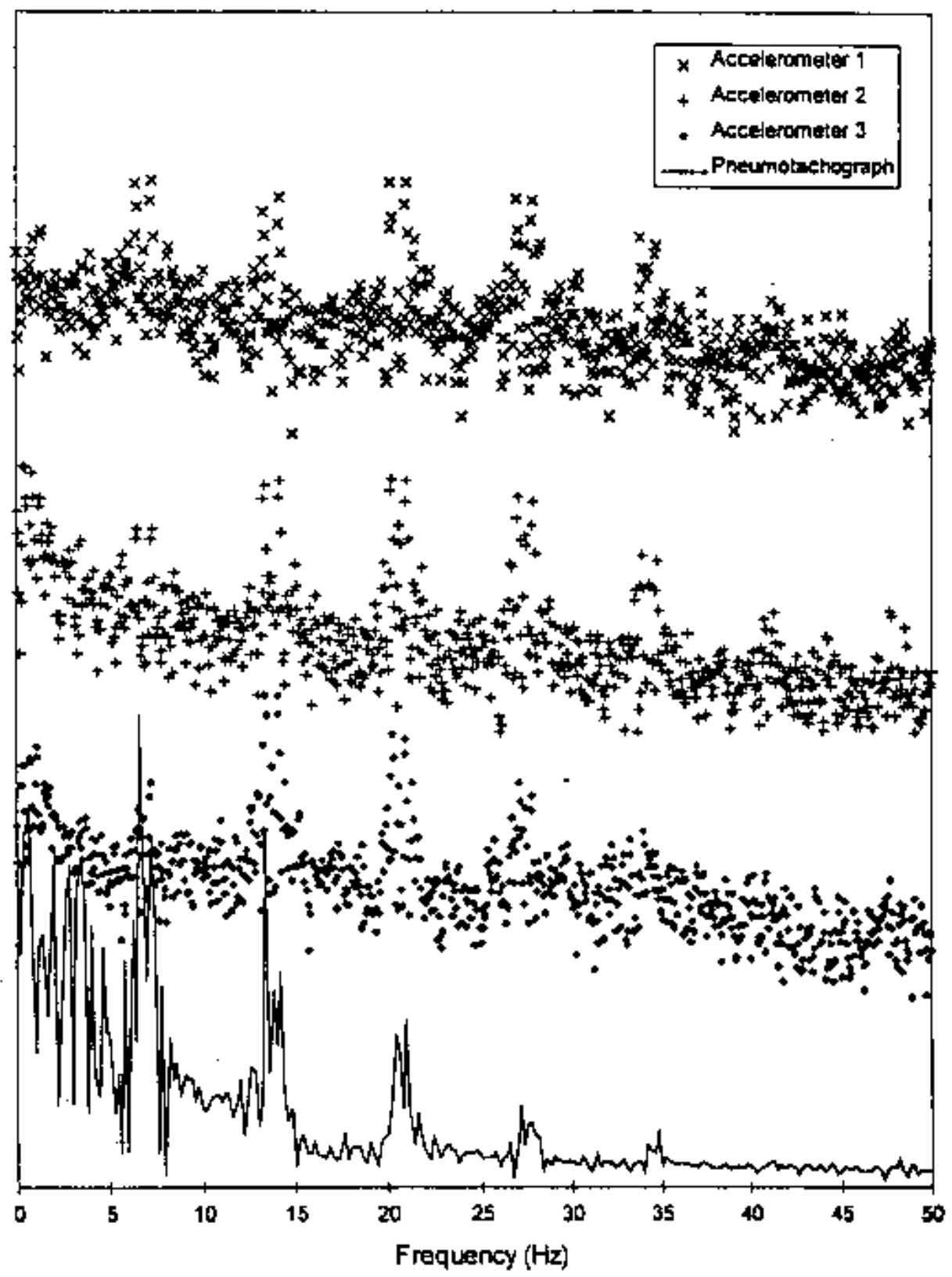

Figure 7. Presence of perturbations on the chest wall: (1) above pectoral $4 \mathrm{~cm}$ right of sternum, (2) below pectoral 4 $\mathrm{cm}$ right of sternum, (3) $2 \mathrm{~cm}$ right of seventh dorsal vertebra 


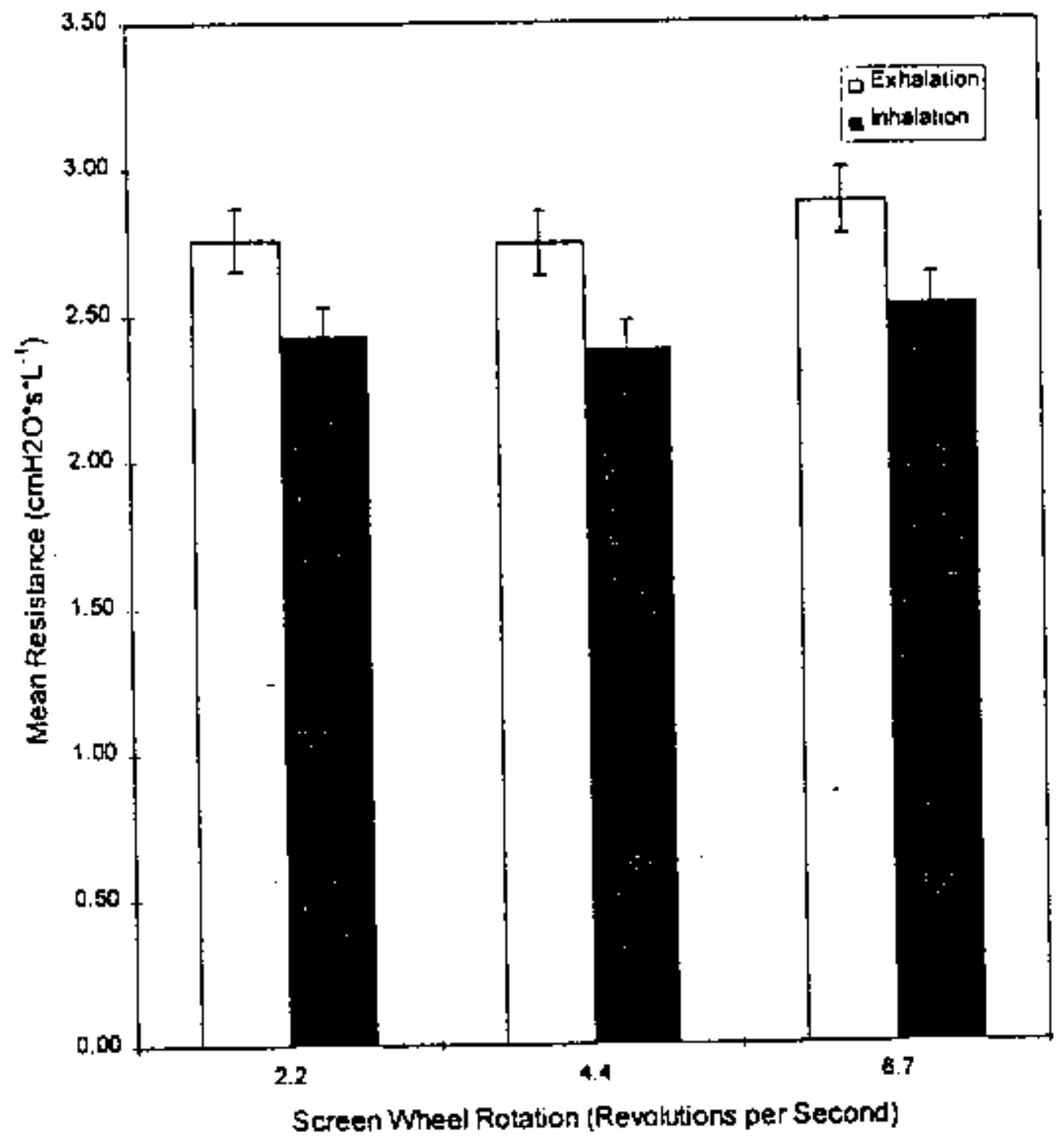

Figure 8. Average APD resistance at three screen-wheel speeds.

Assuming that subject resistance does not change when external resistance is added, slopes would be expected to equal unity. These slopes of less than unity indicate that either the APD is not fully sensitive to resistance increase or that the subject resistance tends to decrease when external resistors are added. There are at least two reasons why resistance may decrease during the test. First, additional pneumotachographs add dead space to the system; $37 \mathrm{ml}$ each. This extra dead space will increase tidal volume and therefore increase average lung volume. The increased lung volume will cause a small decrease in resistance. Second, the added back pressure of the pneumotachograph resistors helps to keep compliant air passages from collapsing during expiration, which may decrease expiratory resistance. While it is clear that the APD does reliably detect increases in system resistance, it remains possible that the magnitude of the increase is underestimated.

\subsection{Comparison of APD and plethysmographic resistance}

Plethysmographic resistance for 20 of the subjects ranged from 0.45 to $1.39 \mathrm{cmH}_{2} \mathrm{O} \mathrm{s} \mathrm{l}^{-1}$, while average APD resistance $\left(R_{\text {avg }}\right)$ ranged from 2.17 to $3.90 \mathrm{cmH}_{2} \mathrm{O} \mathrm{sl}^{-1}$. Inspiratory resistance $R_{\text {avg,in }}$ ranged from 1.91 to $3.71 \mathrm{cmH}_{2} \mathrm{O}$ $\mathrm{s} \mathrm{l}^{-1}$, slightly lower than expiratory resistance $R_{\text {avg,ex }}$ which ranged from 2.43 to $4.54 \mathrm{cmH}_{2} \mathrm{O} \mathrm{s} \mathrm{l}^{-1}$. One subject had a much higher plethysmographic resistance of $2.55 \mathrm{cmH}_{2} \mathrm{O} \mathrm{s} \mathrm{l}^{-1}$ while having an $R_{\text {avg }}$ merely at the high end of the range, $3.71 \mathrm{cmH}_{2} \mathrm{O} \mathrm{s} \mathrm{l}^{-1}$. This subject was not considered for the linear regression shown on figure 10 . For the 20 subjects, there was an $80 \%$ correlation between $R_{\text {aw }}$ and $R_{\text {avg. }}$. Regression analysis shows this relationship to be statistically significant $(p<0.0001)$. 


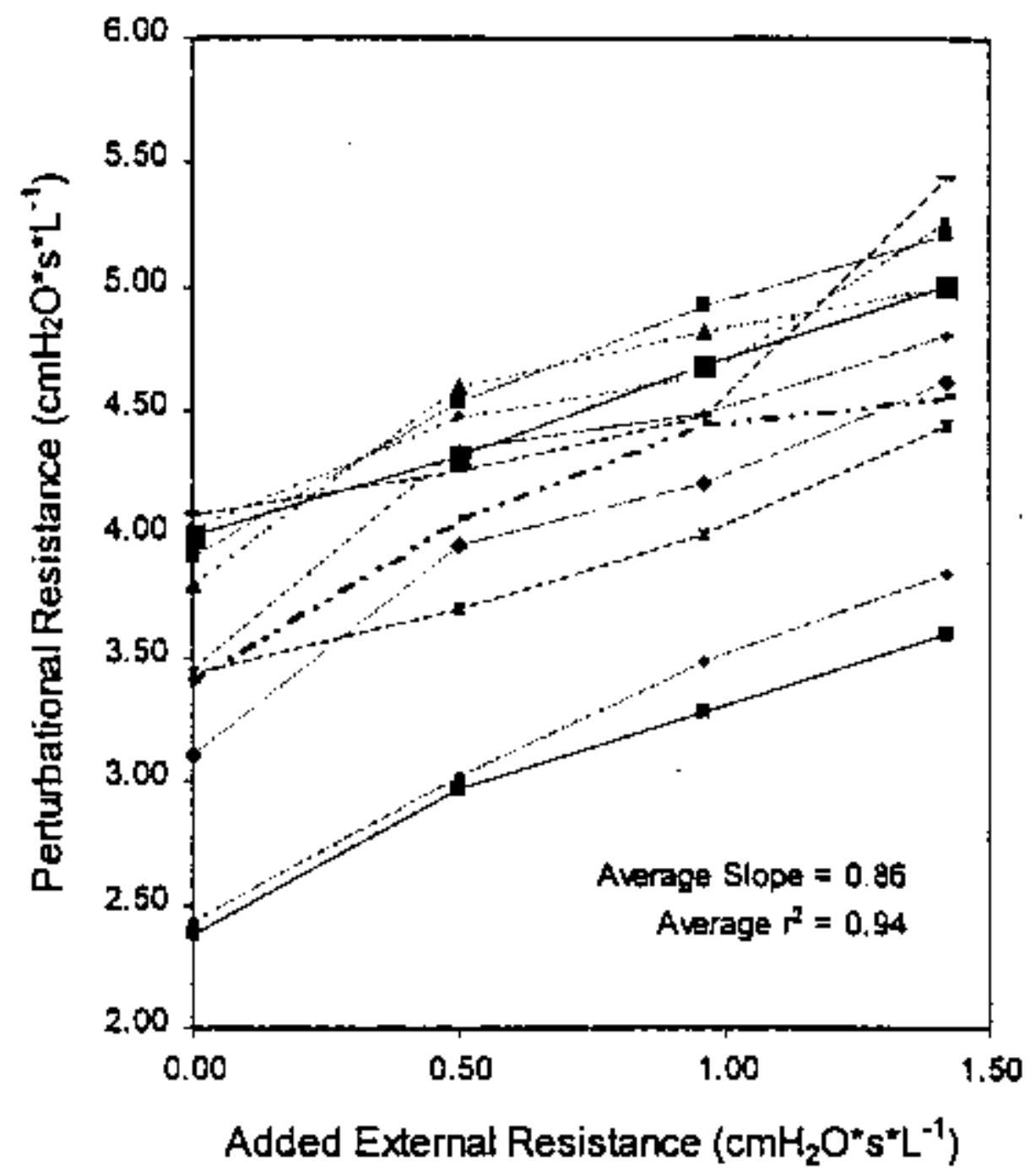

Figure 9. Change in APD-measured expiratory resistance with added externa1 resistance. Dotted lines connect individual subjects. The left-most data point on each line represents baseline resistance.

Table 2. Comparison of perturbational resistance findings.

\begin{tabular}{|c|c|c|c|c|}
\hline Author & $\begin{array}{l}\text { Flow } \\
\text { direction }\end{array}$ & $\begin{array}{l}\text { Peturbational } \\
\text { resistance }\end{array}$ & $\begin{array}{l}\text { Plethysmographic } \\
\text { resistance }\end{array}$ & $\begin{array}{l}\text { Number of } \\
\text { subjects }\end{array}$ \\
\hline Sobol (1971) & Inhalation & $2.0-4.4$ & $0.66-1.77$ & 18 \\
\hline Kures (1974) & Both & $1.2-2.2$ & $1.2-2.0$ & 4 \\
\hline $\begin{array}{l}\text { Schmid-Schoenbein } \\
\text { and Fung (1978) }\end{array}$ & Both & $1.7-3.5$ & & 11 \\
\hline Shaw et al (1983) & Exhalation & $0.6-2.2$ & $0.8-2.5$ & 18 \\
\hline Shaw et al (1983) & Exhalation & $2.2-3.1$ & & 4 \\
\hline Johnson et al (1984) & Both & $0.8-2.0$ & & 5 \\
\hline Lausted (1997) & Both & $2.0-4.0$ & $0.45-2.55$ & 21 \\
\hline
\end{tabular}

There are at least four reasons why the slope of the regression might exceed unity. It is possible that the subjects with higher airway resistance also had higher tissue resistance. It is possible that the body plethysmograph functionally underestimates resistance. This is possible because the plethysmograph does not actually measure alveolar pressure, but rather it measures average thoracic gas pressure and then estimates alveolar pressure. It is also possible that the APD overestimates resistance. Alternatively, the slope might actually be 1.00 and the 1.36 estimate might be due to random error; in this experiment a small number of subjects with a small range of resistance levels were tested. Ninety-five per cent confidence intervals place the slope between 0.85 and 1.88 . 


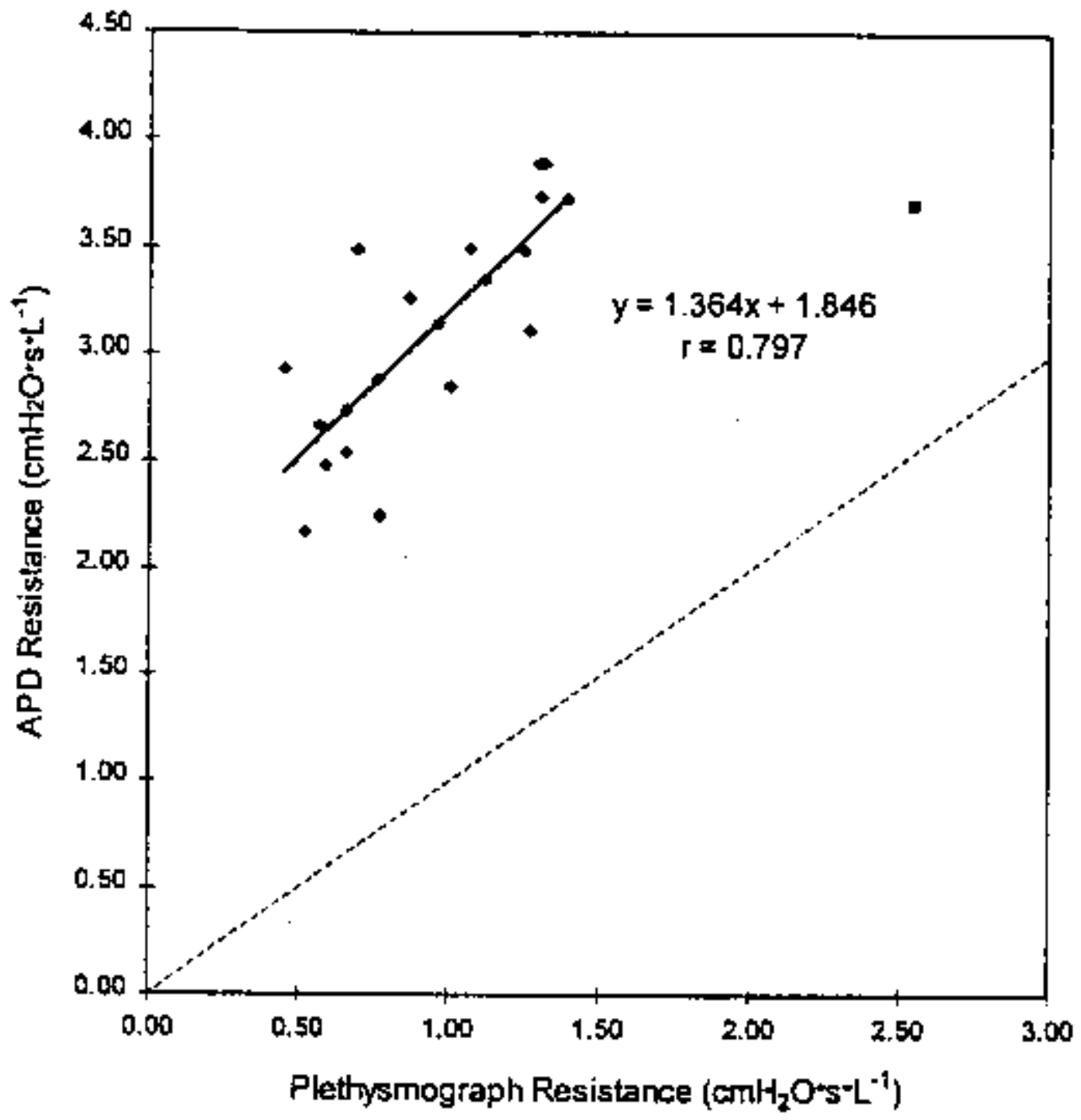

Figure 10. Average perturbational resistance plotted against plethysmographic resistance for 21 normal subjects. The broken line is the line of identity.

\section{Discussion}

Other authors have tested healthy adult subjects with perturbational techniques. The range of values reported here are similar to those reported in 18 subjects by Sobol (1971) and in 11 subjects by Schmid-Schoenbein and Fung (1978). A lower range of values was reported in five subjects by Kures (1974) and in five subjects by Johnson et al (1984). Shaw et al (1983) reported a lower range of values in 18 subjects who were tested for both perturbational resistance $\left(R_{\mathrm{apd}}\right)$ and $R_{\mathrm{aw}}$ and a comparable range of values for four subjects for whom $R_{\mathrm{aw}}$ was not given. Kures and Johnson et al explain their low values by stating that $R_{\text {apd }}$ is not respiratory resistance but airway resistance. Both assume that perturbations do not extend past the alveolar level. Johnson and Lin (1983b) theorize that tissue compliance dampens the perturbation so it becomes negligible before reaching the alveolar level. This would mean that $R_{\text {apd }}$ is less than $R_{\text {aw }}$; that $R_{\text {apd }}$ is more determined by upper airway resistance than lower airway resistance. Shaw et al do not have an explanation for their low $R_{\text {apd }}$ values. They expected $R$ to exceed $R_{\text {aw }}$ by the magnitude of tissue resistance.

There are at least two reasons why reported resistance ranges would vary. One reason is subject variability. Four subjects tested by one group could all have respiratory resistance levels lower than four subjects tested by another group. Another difference may be due to the differing implementation of perturbational techniques. For example, Sobol and Shaw et al did not measure $\Delta p_{m}$. in their calculations, but calculated it as screen resistance $\left(R_{\mathrm{scr}}\right)$ multiplied by $\Delta V^{\prime}$. This introduces an error since $R_{\text {scr }}$ is not constant; it increases with flow. This would cause $R_{\text {apd }}$ to be underestimated if $R_{\text {scr }}$ is calibrated at a flow less than the flow at which the perturbation occurs. A better value of $R_{\text {apd }}$ is found by using both $\Delta p_{\mathrm{m}}$ and $\Delta V^{\prime}$. 
Other authors have observed a correlation between perturbational resistance and $R_{\text {aw }}$ (table 2). Their studies have included children, asthmatics and pulmonary disease patients in order to expand the range of resistance levels. Shaw et al (1983) concluded that their normal subjects had $R$ values within $25 \%$ of $R_{\text {aw }}$ values and that 14 COPD (chronic obstructive pulmonary disease) patients, representing a range of resistance levels up to $7 \mathrm{cmH}_{2} \mathrm{O} \mathrm{sl}^{-1}$, had $R$ values within $10 \%$ of $R_{\text {aw }}$ values. Kures (1974) stated that his $R$ and $R_{\text {aw }}$ values were comparable, based on six asthmatic children and nine healthy children in addition to his four healthy adults. The children represented a range of resistance levels up to $5 \mathrm{cmH}_{2} \mathrm{O} \mathrm{s} \mathrm{l}^{-1}$. Sobol (1971) compared his $R_{\text {apd }}$ with $R_{\text {aw }}$ in 82 respiratory disease patients in addition to his 18 healthy subjects and found a $77 \%$ correlation. Values ranged up to 16 and $10 \mathrm{cmH}_{2} \mathrm{O} \mathrm{s} \mathrm{l}^{-1}$ respectively.

The technique used in the present study improves upon those used in previous studies. The measurements are less subjective. Four of the previous studies saved flow and pressure data tracings with a strip chart recorders for later analysis by hand. The detection of perturbations and the measurement of their magnitude was done by eye. The data collected by Schmid-Schoenhein and Fung (1978) was saved digitally, and the analysis computer-aided, but the detection of perturbations was still done by eye. The present study minimized operator bias in the determination of $R_{\text {avg. }}$. Data were collected digitally. Perturbations were detected by computer. Only perturbations with small pressure or flow magnitudes below a preset threshold were rejected. $R_{\text {apd }}$ values were calculated from all other perturbations.

\section{Conclusions}

The overall conclusions for this study, then, are that the APD can be used to make respiratory resistance measurements that are consistent and sensitive to changes. With its compact size, light weight, rapid measurements and separation of inhalation and exhalation resistances, the APD is an instrument that can be useful in a pulmonary function laboratory.

\section{References}

Barnas G M. Yoshino K, Loring S H and Mead J 1987 Impedance and relative displacements of relaxed chest wall up to $4 \mathrm{~Hz}$. J. Appl. Physiol. 62 71-81

DuBois A B, Botelho S Y and Comroe J H 956 A new method for measuring airway resistance in man using a body plethysmograph: values in normal subjects and in patients with respiratory disease $J$. Clin. Invest. 35327 35

Johnson A T 1986 Conversion hetween plethysmograph and perturbational airways resistance measurements IEEE Trans. Biomed. Eng. 33 803-6

Johnson A T. Berlin H M and Purnell S A 1974 Perturbation device for noninvasive measurement of airway resistance (abstract) Med. Instrumen. 8141

Johnson A T and Lin C S 1983a Airflow perturbation device for measuring airways resistance of animals Trans. ASAE 26 503-6

-1983b Airflow resistance or conscious boors Trans. ASAE 26 1150-2

Johnson A T, Lin C S and Hochheimer J N 1984 Airflow perturbation device for measuring airways resistance of humans and animals IEEE Trans. Biomed Eng 9 622-6

Johnson A T and Milano J M 1987 Relation between limiting exhalation flow rate and lung volume IEEE Trans. Biomed.Eng. 34 257-8

Kures H 1974 An additive method for airway resistance measurement Acta Paediatr Scand. 63 351-6

Lausted C G 1997 Development of the airflow perturbation device MS Thesis University of Maryland, College Park

Lehtola P 1986 Improving the airflow perturbaton device MS Thesis University of Maryland, College Park

Lin C S Johnson A T and Yaramanoglu M 1985 Model analysis or the airflow perturbation device Innov. Technol Biol. Med. 6 461-72

Michaelson E D, Grassman E D and Peters W R 1975 Pulmonary mechanics by spectral analysis of forced random noise J. Clin. Invest. 56 1210-30 
Nagels J, Landser F J, Vander Lindar L, Clement J and Van de Woestijne K P 1980 Mechanical properties of lungs and chest wall during spontaneous breathing J. Appl. Phys. 49 408-16

Schmid-Schoenbein G W and Fung Y C 1978 Forced perturbation of respiratory system Ann. Biomed Eng. 6 194211

Shaw C F, Chiang S T, Hsieh Y C, Milic-Emili J and Lenfant C 1983 A new method for measurement of respiratory resistance J. Appl. Physiol. 54 594-7

Sobol B J 1970 A simplified approach to the measurement of total respiratory impedance Am. Rev Resp. Dis. 102 280-1

—1970b A simple, rapid technique for assessing airway resistance during quiet breathing Am Rev Resp. Dis. 102 970-4

-1971 Clinical experience with a new test of pulmonary function Chest 60 137-41

Stanescu D 1991 Was it just our problem, or yours too? Errors in body plethysmography, in infants, children and adults Pediatr. Pulmonol. 11 285-8

Suki D, Peslin R. Duvivier C and Farre R 1989 Lung impedance in healthy humans measured by forced oscillations from 0.01 to $0.1 \mathrm{~Hz}$ J. Appl. Physiol. $671623-9$

Teculescu D B, Bohadan A B, Peslin R, Pino J and Jansen da Silva J M 1982 Variability, reproducibility, and observer difference of body plethysmographic measurements Clin. Physiol. 2 127-38

Zerah F, Lorino A, Lorina H, Harf A and Macquin-Mavier E 1995 Forced oscillation technique versus spirometry to assess bronchodilatation in patients with asthma and COPD Chest 108 41-7 